\title{
The Emergence of Polychronous Groups under Varying Input Patterns, Plasticity Rules and Network Connectivities
}

\author{
Joseph Chrol-Cannon, André Grüning and Yaochu Jin \\ Department of Computing \\ University of Surrey \\ Guildford, Surrey, GU2 7XH \\ United Kingdom \\ Email: \{j.chrol-cannon, andre.gruning, yaochu.jin\}@surrey.ac.uk
}

\begin{abstract}
Polychronous groups are unique temporal patterns of neural activity that exist implicitly within non-linear, recurrently connected networks. Through Hebbian based learning these groups can be strengthened to give rise to larger chains of spatiotemporal activity. Compared to other structures such as Synfire chains, they have demonstrated the potential of a much larger capacity for memory or computation within spiking neural networks. Polychronous groups are believed to relate to the input signals under which they emerge.

Here we investigate the quantity of groups that emerge from increasing numbers of repeating input patterns, whilst also comparing the differences between two plasticity rules and two network connectivities. We find - perhaps counter-intuitively - that fewer groups are formed as the number of repeating input patterns increases. Furthermore, we find that a tri-phasic learning rule gives rise to fewer groups than the 'classical' double decaying exponential STDP plasticity window. It is also found that a scale-free network structure produces a similar quantity, but generally smaller groups than a randomly connected ErdösRényi structure.
\end{abstract}

\section{ACKNOWLEDGEMENTS}

André Grüning is supported in part by EPSRC grant EP/I014934/1.

\section{INTRODUCTION}

Hebb proposes [1] that cognitive processing was mediated by synaptic plasticity encoding patterns of stimulation in neural networks which could subsequently be re-activated by internal cues. Formally described neuro-plasticity rules, now known as spike-timing dependent plasticity (STDP), express that causal correlations in pre- and post- synaptic activity are potentiated, while a-causal correlations are depressed [2], [3], [4].

Hebb's enduring work led to the study of transient, dynamic patterns of activity and to the definition of neural structures that could underpin them. Abeles [5], describes 'synfire chains' as pools of neurons activating synchronously between connections of fairly equal delays. Bienenstock [6] modifies this concept by suggesting that the delays between each pool do not need to be the same and that a chain can still propagate without the pools activating coherently.
Polychronous groups (PGs) were introduced by Izhikevich [7] as an alternative to viewing spiking computation as synchronously activating chains of neurons. Given a specific input, a time-locked but crucially - temporal - response can be reproduced when a given PG 'fires'. The advantage this provides is that the same neurons could easily be part of a huge multitude of different groups, drastically increasing the potential capacity of even small networks well beyond their size in terms of the number of synapses.

Izhikevich [7] observes the capacity of a network to support PGs by providing continuous Poissonian input to randomly selected neurons. Thousands of PGs are observed to arise, but due to the unstructured input, the groups capacity to internalize responses to fixed input patterns is unclear.

Here, we investigate the capacity for PGs when given a number of fixed spatiotemporal input patterns repeated over the course of the simulation. We do this by presenting increasing numbers of inputs to a recurrently connected network of Izhikevich neurons [8] and monitoring the number of groups generated as a result.

As well as the number of inputs playing a role in the PGs formed, the synaptic plasticity rule is central too. Here we choose to compare two types of learning windows; the 'classical' double decaying exponential rule used previously by Izhikevich [7] and a tri-phasic rule observed in CA3-CA1 hippocampal synapses [4].

In his simulations pertaining to PGs [7], Izhikevich forms the connections between neurons randomly and independently of existing connections, giving rise to an Erdös-Rényi structured network. We compare this to a scale-free topology that has been widely adopted within the field of reservoir computing [9].

\section{MethodS}

\section{A. Network}

A network of 1000 Izhikevich neurons [8] is randomly connected with a probability of any two neurons being connected as 0.1 . The ratio of excitatory to inhibitory neurons is $4: 1$. Excitatory weights are initialized as 6.0 and inhibitory 
weights as -5.0. The maximum weight value is 10.0. Excitatory delays are randomly drawn from a uniform distribution up to $20 \mathrm{~ms}$ while inhibitory delays are set at $1 \mathrm{~ms}$. The simulation timestep is $1 \mathrm{~ms}$. Synaptic transmission occurs as a single impulse lasting $1 \mathrm{~ms}$.

\section{B. Connectivities}

Two widely known connectivity graph types are used:

Erdös-Rényi graph is the simplest - to add an edge, nodes are selected at random. Each new edge is added independently to all pre-existing ones.

Scale-Free graph is more structured around 'hubs' of highly connected areas. To add an edge, the probability of selecting a node as a source is proportional to its out-degree while the target neurons are selected without bias to help maintain full network utilization.

\section{Plasticity Rules}

Weight changes are calculated every $1 \mathrm{~ms}$, but accumulated for $1 \mathrm{~s}$ and then applied. Synaptic plasticity is governed according to STDP and the learning windows described in 1. Only excitatory connections undergo learning, with inhibitory weights remaining fixed at their initial values. The minimum weight is 0 for excitatory neurons. A nearest-neighbor STDP approach is taken with only the most recent spikes being considered to calculate the weight change [10].

Classic STDP is depicted in graph 1a. as the familiar double decaying exponential with Long Term Depression (LTD, negative) and Long Term Potentiation (LTP, positive) sections divided by a discontinuity. This rule has been observed in both the neocortex and cerebellum as well as other areas of the brain [2], [3], [4]. For the classic STDP rule we use the following equation:

$$
\Delta W(t)= \begin{cases}A_{+} \cdot e^{-\Delta t / \tau_{+}} & \text {if } t>0 \\ A_{-} \cdot e^{\Delta t / \tau_{-}} & \text {if } t \leq 0\end{cases}
$$

with the paramters: $A_{+}=0.1, A_{-}=-0.12, \tau_{+}$and $\tau_{-}$are 20.0, that match Izhikevich's STDP model.

Tri-phasic Rule is a continuous function as depicted in graph 1b. It was introduced to match synaptic strength changes as observed between the CA3 and CA1 areas of the hippocampus [4]. The tri-phasic plasticity rule that we use is shown by:

$$
A_{+} \cdot e^{-(x-15)^{2} / \tau_{+}} \quad-\quad A_{-} \cdot e^{-(x-20)^{2} / \tau_{-}}
$$

with the paramters: $A_{+}=0.23, A_{-}=0.15, \tau_{+}=200$, and $\tau_{-}=2000$. These values were chosen to match the 'classical' STDP rule in: the total duration of the learning window, the area of integration under the curves and therefore the overall levels of LTP and LTD.

\section{Input Patterns}

The idea when generating the input patterns is that they should all follow the same distribution of spikes and average rate while differing only in the precise spike timing. This is because polychronous groups are sensitive to precise timings and we can rule out variation in the number of groups due to changes in the input rate - only the number of different patterns presented counts. Input patterns are provided as pre-calculated Poisson spike trains with a $10 \mathrm{~Hz}$ rate and a minimum 5ms Inter-Spike-Interval left after each spike. Each pattern consists of 100 spike trains fed to 100 pre-selected neurons chosen at the beginning of the simulation. Each pattern lasts a whole second and is applied from the beginning of a second. A set of 1,2, 4 or 8 patterns are repeatedly alternated throughout the duration of the simulation.

\section{E. $P G$ Detection}

In [7], Izhikevich outlines a definition of what constitues a polychronous group and its main characteristics. It is this that we base our detection process on. The key idea is that for a given excitatory neuron (root), any triplet of pre-neurons with strong connections (anchors) may be the beginning of a long chain of propagation. There are two factors that determine whether a group will then arise. Firstly, the anchors should fire at times such that the spike events reach the root at the same time, thus causing a new spike. Secondly, there should be further strong connections from the anchors and the root to more neurons that can continue to propagate spikes.

In order to detect all the neurons that form a group, a simulation approach is taken. For each set of anchors that are stimulated at the appropriate times, the whole network is simulated to allow other neurons to fire. This simulation must be started on an otherwise silent network (one with no other activity) so that we can tell that all of the spikes that occurred were a result of the original anchors firing. The resultant propagation of spikes forms a potential group. Small groups are discounted from the detection procedure. The path length of a group is the longest chain of connections from the first to last firing. For the purpose of ignoring tiny groups, this path length should be at least seven links long for a group to be considered. On the other hand, because the simulation time is limited to 150 milliseconds for performance reasons, larger groups could be truncated - still counted, but not registered as their full size.

Figure 2 illustrates a minimal PG with longest path length equal to seven, and with each anchor neuron contributing to at least two spikes.

\section{RESULTS}

\section{A. Effect of the Number of Input Patterns on Group Emergence}

It may be expected that the more input patterns presented to the network, the more groups are reinforced and detected over time. Actually the opposite was observed.

From the averaged results of ten independent runs, Figure 3 shows starkly that as the number of presented input patterns doubles, the resultant number of groups formed roughly halves.

We suggest that the cause for this is due to each pattern re-reinforcing a specific set of connections throughout the majority of the network, thus forming groups. At the same time, different patterns undermine each others groups by 

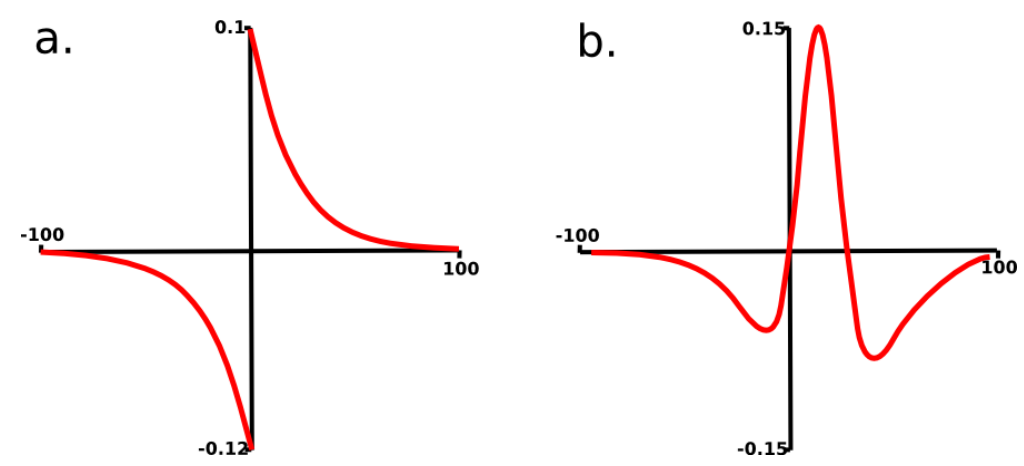

Fig. 1. The two plasticity rules used in our simulations. Graph a. shows the classical double decaying exponential STDP rule. Graph b. shows a more recently discovered tri-phasic rule.

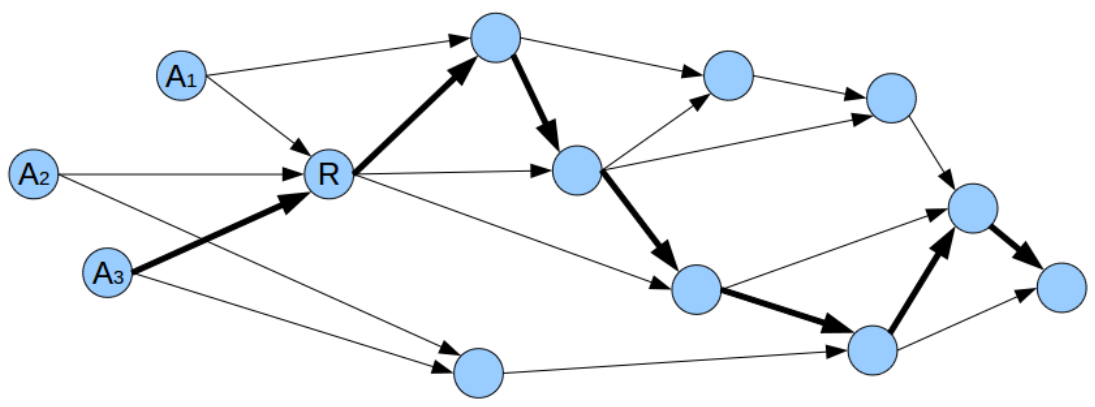

Fig. 2. Illustration of a minimal polychronous group. The anchor neurons are labeled with 'A' and the root neuron with 'R'. Bold connections highlight a longest path length for this group that happens to be seven links long. Each anchor neuron connects to the root and at least one other neuron.

causing a recently strengthened set of connections to fire outof-order in relation to how a previously presented pattern had caused. This could be an example of competition between the inputs leading to a forgetting effect similar to the one observed in [11].

The key point is that each pattern presented to the network causes specific temporal activity throughout most of the network meaning that with just one input, most of the connections will undergo learning relevant to that input, forming groups throughout as a response.

In this light, any further pattern presented afterwards is only likely to disrupt the previously formed groups while strengthening new ones. Contention over the synapses is evident amongst different inputs - the more inputs, the greater the contention, the fewer groups can ultimately form. To view things in a selectionist way: perhaps only the strongest groups (enforced by any given pattern) can survive.

\section{B. Effect of the Plasticity Rule on Group Emergence}

Clearly, from a comparison between the two plasticity rules in Figure 3(a) and 3(b), the decaying exponential STDP gives rise to a much larger number of groups than the tri-phasic STDP - about eight times as many.

The big difference is probably due to the consistency of the caused weight changes. With the traditional rule, there are only two modes of change: early and late. If a spike arrives early before a firing is caused, efficacy is strengthened; and if it arrives late it is weakened. This means that regardless of the delay, as long as connected neurons always fire in the same order, the weight change will be in a consistent direction.

On the other hand, the tri-phasic rule has three modes; early, late and on-time. Only on-time delivery will increase synaptic efficacy here, therefore even if connected neurons mostly fire in the same order, the difference of how early or late they are could cause opposing weight change.

In a similar way to the high contention caused by a larger number of input patterns, a more stringent criterion provided by the tri-phasic rule may lead to a more challenging selection process for polychronous groups.

Rather than being a disadvantage in terms of reduction in capacity, the emphasis on selection of the fittest groups for each pattern may present advantages when viewing the neural microcircuit less as a storage vessel, and more like a selectional system, refer to Edelman [12].

\section{Effect of Network Connectivity on Group Emergence}

It is found that switching the network graph type from Erdös-Rényi to Scale-Free does not have a significant effect on the number of groups detected, but it does reduce the average size. This is evident by comparing the histograms: Figure 4(a) and Figure 4(b) - group size distributions from Erdös-Rényi and Scale-Free graphs, respectively. For each histogram, 2000 groups were sampled over the duration of the simulation.

Due to the Erdös-Rényi connectivity model being more 

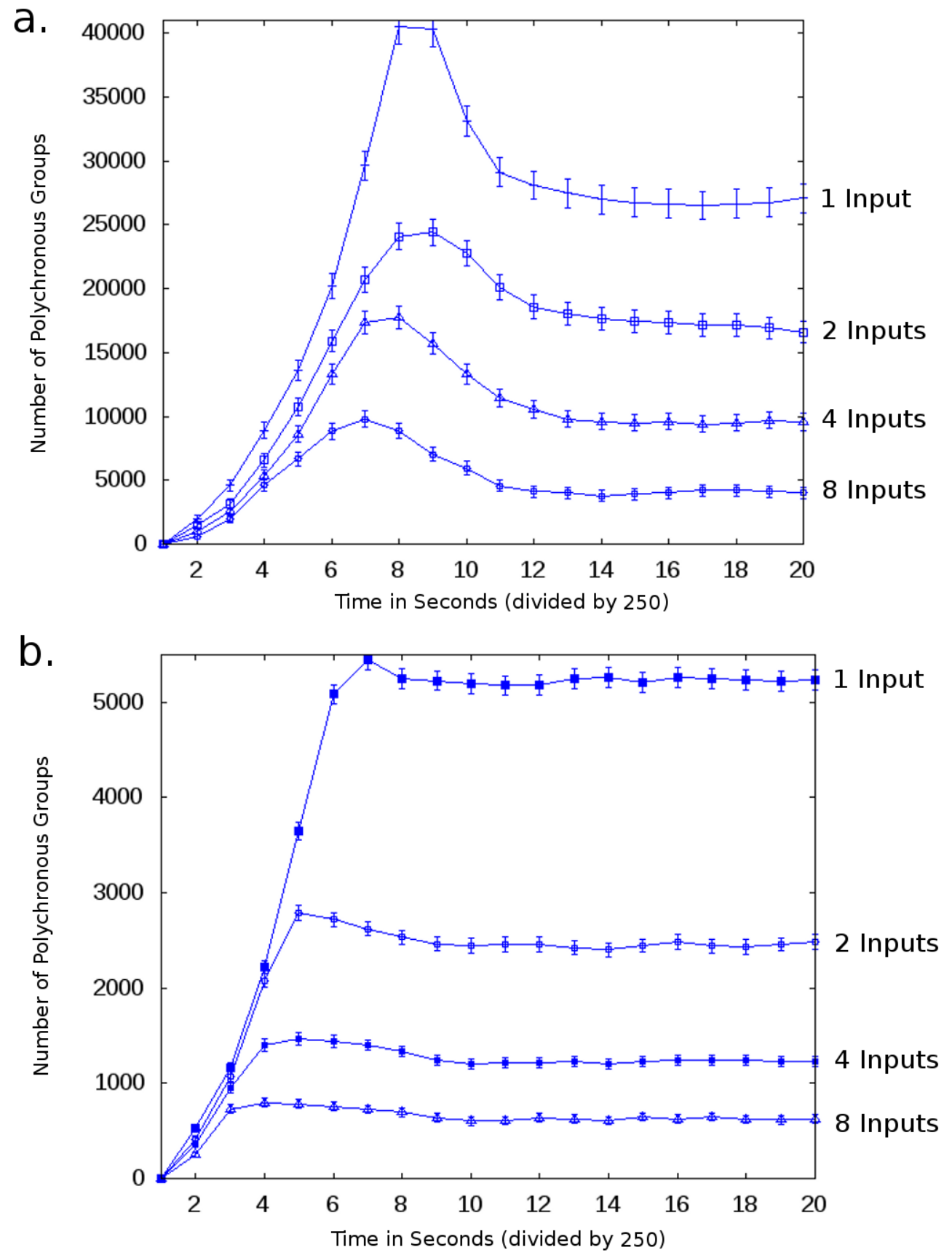

Fig. 3. Emergence of groups over a 5,000 second time period while being stimulated by; 1, 2, 4 or 8 alternating patterns. Snapshots of numbers of groups within the network are taken at 250 second intervals. a) shows the simulation subject to the 'classical', decaying exponential STDP rule. b) shows the simulation subject to the CA1 type, tri-phasic STDP rule. Ten simulation runs were performed for each set of results and averaged to produce the two graphs. Error bars are shown as $3 \mathrm{x}$ SD of each sampling point.

inclusive of any neurons than the Scale-Free model, it is not surprising that for a given stimulus, a larger portion of the networks neurons would fire. However, as PGs are based on unique combinations of strong pre-synapses - and both connectivity models are allowed the same number of synapses, governed by the same plasticity rules - the total number of PGs is fairly even between them.
The difference can be summarized as larger, more spread out PGs for Erdös-Rényi graphs and smaller, more localized PGs for Scale-Free graphs. When it comes to the wider utility of polychronous groups, it is not clear which of size or localization is more desirable. 
a.

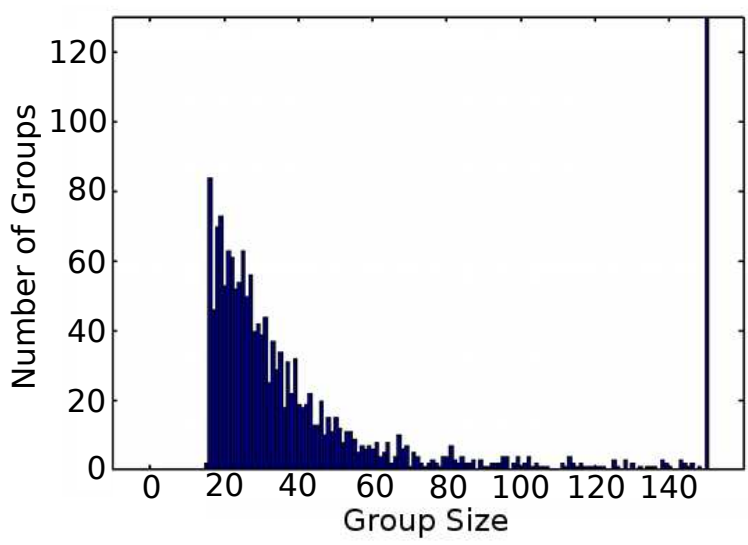

b.

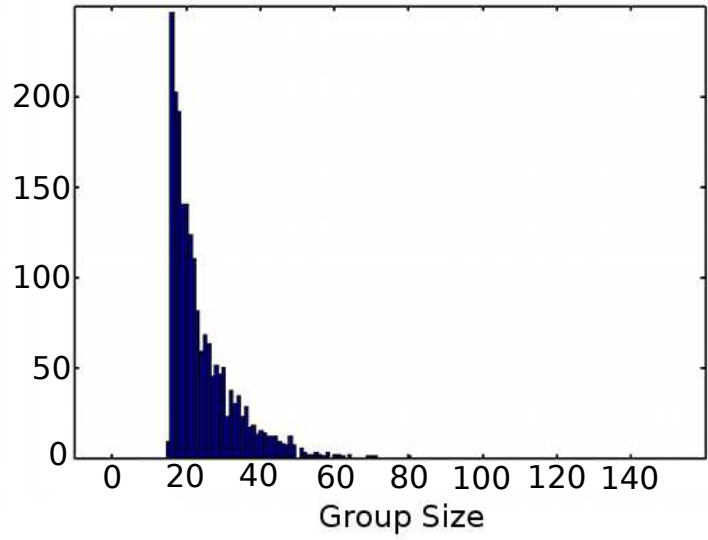

Fig. 4. Histograms of group sizes from 2000 samples of each network structure: a. - Erdös-Rényi. b. - Scale-Free.

\section{Discussion OF RELATED WORK}

\section{A. Other Definitions of PGs}

In [13], Martinez and Paugam-Moisy provide a clearer and perhaps a more general definition of a PG than has been given previously. A PG consists of a temporal chain of potential activation, not just the neurons that constitute the group. The propagation of activity is started by a small number, $s$, of spikes from triggering neurons arriving close enough together in time to cause further spikes.

Three types of group are distinguished:

Supported PGs : Based soley on the network topology.

Adapted PGs : Synaptic weights and neuron membrane model are also considered.

Activated PGs : Groups that are actually observed to occur.

In our analysis of PG capacity, we are detecting Adapted PGs as they are the groups directly subject to change under STDP learning rules.

\section{B. PGs Analysed in Learning Task}

In [14], Paugam-Moisy and Martinez use PGs to help analyse the separability of their network on a two class classification problem.

Due to fairly low network connectivity, only 104 supported PGs existed in total. STDP is the only adaptation process within the network (delay learning rule being applied to chosen outputs).

Activated PGs were detected during both learning and testing phases and analysed in order to ascertain the specificity of PGs to the two classes in a classification problem. Small subsets of PGs are observed specific to each of the two patterns. Also, the most frequently activated groups in the testing phase are also the ones observed during the learning phase. This supports the idea that the groups are representative of the two encoded classes.

\section{CONCLUSION}

The results have shown how the PG capacity of a network is reduced when the number of inputs given increases. This may not be a problem in the trials that we performed as a significant number of PGs still did form.

Although the tri-phasic rule generates fewer PGs than the 'classical' STDP rule, the smaller error bars in Figure 3a over Figure $3 b$ show them to be significantly more stable. The variance between the ten simulation runs being up to ten times greater in Figure 3a than in Figure 3b. This could indicate an interesting trade-off between capacity and stability in the areas of the brain which use different plasticity rules. Brain functions that require a larger number of responses for short term use may favour the 'classical' rule, while functions that require fewer responses but with longer term stability may favour the tri-phasic rule.

Further work could add strength to a number of conclusions:

Testing larger networks with weaker synapses could reduce the competition between input patterns over the same connections. Given a large enough network, competition could be reduced to the extent that increasing the number of spatiotemporal input patterns may not reduce the number of groups formed - at least up to a certain limit.

Testing much larger numbers of input patterns would enable us to see when the number of PGs that form dwindles to the point that there are insufficient numbers of PGs to internalize all of the inputs presented to the network. This would allow stronger statements to be made on catastrophic forgetting in a neural system based on PGs.

Analyzing the specificity of the input patterns to the PGs that they activate would enable confirmation that particular groups are uniquely internalizing the inputs. This would demonstrate the level of seperability that the PGs provide and also allow statements to be made on cross-talk in the responses between the inputs rather than limiting the discussion to a more general level of competition.

Analyzing group stability by scanning for individual groups 
throughout the simulation period to determine persistence. In terms of stability of group activation, some noise could be added to each input pattern to discern each groups resilience to noise.

\section{REFERENCES}

[1] D. Hebb, The Organization of Behaviour. Wiley, 1949.

[2] G. Q. Bi and M. M. Poo, "Synaptic modifications in cultured hippocampal neurons: dependence on spike timing, synaptic strength, and postsynaptic cell type," Journal of Neuroscience, vol. 18(24), pp. 10 464-72, 1998.

[3] H. Markram, J. Luebke, M. Frotscher, and B. Sakmann, "Regulation of synaptic efficacy by coincidence of postsynaptic aps and epsps," Science, vol. 275(5297), p. 2135, 1997.

[4] G. M. Wittenberg and S. S. Wang, "Malleability of spike-timingdependent plasticity at the ca3-ca1 synapse," Journal of Neuroscience, vol. 26, pp. 6610-6617, 2006.

[5] M. Abeles, Corticonics: Neural circuits of the cerebral cortex. Cambridge: Cambridge University Press, 1991.

[6] E. Bienenstock, "A model of neocortex," Network: Computation in Neural Systems, vol. 6, pp. 179-224, 1995.

[7] E. M. Izhikevich, "Polychronization: Computation with spikes," Neural Computation, vol. 18, pp. 245-282, 2006.

[8] — - "Simple model of spiking neurons," IEEE Transactions on Neural Networks, vol. 14, pp. 1569-1572, 2003.

[9] W. Maass, T. Natschlaeger, and H. Markram, "Real-time computing without stable states: A new framework for neural computation based on perturbations," Neural Computation, vol. 14, pp. 2531-2560, 2002.

[10] E. M. Izhikevich, "Relating stdp to bcm," Neural Computation, vol. 15, pp. 1511-1523, 2003.

[11] C. Fernando, R. Goldstein, and E. Szathmary, "The neuronal replicator hypothesis," Neural Computation, vol. 22(11), pp. 2809-2857, 2010.

[12] G. M. Edelman, Neural Darwinism: The theory of neuronal group selection. New York: Basic Books, 1987.

[13] R. Martinez and H. Paugam-Moisy, "Algorithms for structural and dynamical polychronous groups detection," in Proc. of Int. Conf. on Artificial Neural Networks ICANN'09, vol. 5769, 2009, p. 7584.

[14] H. Paugam-Moisy, R. Martinez, and S. Bengio, "Delay learning and polychronization for reservoir computing," NeuroComputing, vol. 71, pp. 1143-1158, 2008. 